\title{
NUMERICAL ANALISYS OF GUIDED ULTRASONIC WAVE PROPAGATION IN FIBER METAL LAMINATES
}

\author{
Andrey Mikhaylenko ${ }^{1 *}$, Nanda Kishore Bellam-Muralidhar ${ }^{2}$, Natalie Rauter ${ }^{1}$, \\ Dirk Lorenz ${ }^{2}$ and Rolf Lammering ${ }^{1}$ \\ ${ }^{1}$ Helmut-Schmidt-University / University of the Federal Armed Forces Hamburg, \\ Holstenhofweg 85, 22043 Hamburg, Germany \\ 2 Technische Universität Braunschweig, Universitätsplatz 2, 38106 Braunschweig, Germany \\ *mikhayla@hsu-hh.de
}

Fiber metal laminates (FML) combine the ductile properties of metal with the high specific stiffness of fiber reinforced plastics. FML also offer substantial reduction in weight along with excellent fatigue strength. These features of FML lead to a dramatic rise of interest in such materials for aeronautical structures lately. However, one of the most vulnerable failures for FML is impact-related delamination which is not detectable with the naked eye. Such damage has to be detected in time to enable a possible repair. Structural health monitoring with the guided ultrasonic waves (GUW) could potentially serve the purpose of damage detection in thin structures by using the physical phenomena of wave propagation interacting with structure defects [1].

The focus of this work is on the numerical simulation of GUW propagation in FML structures. The investigation of this subject follows as forward and inverse problem analysis. Based on an already existing 2D model [2] a 3D finite element model is developed using COMSOL Multiphysics ${ }^{\circledR}$ Software involving the excitation of waves and observing its propagation in the structure. One crucial aspect here is the model discretization and hence, the corresponding element size. To validate the numerical model the wave propagation and the resulting displacement field are compared to the analytical solution derived from the dispersion relation. In this context a mode selective excitation is used in order to have a clear observation and to be able to separate different wave modes.

Furthermore, a model-based inverse solution in a stochastic framework is described for the damage detection, localization and characterization with the aid of a simpler case study on 2D elastic wave equation. The inference problem uses a projection-based parameterized reducedorder model rather than the high fidelity model in order to significantly reduce the computational costs.

\section{References}

[1] R. Lammering, U. Gabbert, M. Sinapius, T. Schuster, P. Wierach (Eds)(2018) Lamb-Wave Based Structural Health Monitoring in Polymer Composites, Springer International Publishing, Cham.

[2] L. Rittmeier, T. Losch, M. Sinapius and R. Lammering (2018) Investigation on the influence of material interfaces and impedance changes on the propagation of guided waves in laminated steel layers. Procedia Manufacturing, 24, 196-202. 